\title{
Barreras, brechas e inequidades de género en trabajos masculinizados en una empresa minera del norte de Chile*
}

* Fecha de recepción: 15 de agosto de 2015. Fecha de aceptación: octubre de 2016.

\section{Resumen}

El siguiente artículo expone los resultados de una investigación en la que uno de sus propósitos fue conocer las características de la llamada "empresa masculinizada", representada por una minera de la ciudad de Copiapó de Chile y cómo, en función de aquello, dicha empresa produce y reproduce estereotipos, roles e identidades de género, desarrollando brechas, barreras e inequidades para el trabajo de las mujeres.

El estudio mantuvo una perspectiva cualitativa y permitió, a través de entrevistas en profundidad, la develación de discursos sometidos a análisis de contenido.

Las conclusiones plantean segregaciones horizontales y verticales de género; la construcción social de los cuerpos de las mujeres; y las restricciones intrínsecas y extrínsecas de género para las mujeres en el marco del trabajo en la minera, así como preguntas y desafíos que como sociedad nos tenemos que hacer para avanzar en una igualdad de género y justicia social.

\footnotetext{
Abstract

The following article presents the results of an investigation where one of its purposes was to know the characteristics of the "masculinized company" represented by a mining in Copiapó City, Chile. And how this company produces and reproduces stereotypes, gender roles and identities, developing gaps, barriers and inequities for women's work.
}

Palabras clave:

identidades, roles y estereotipos de género

Keywords:

identities, roles and gender stereotypes 
1 Nos referiremos a "la Minera" dado que existe un acuerdo, con trabajadores/as y gerentes/as, de confidencialidad del nombre de la empresa.
The study adopted a qualitative perspective, through in-depth interviews, speeches unveiling subjected to content analysis.

The findings raise horizontal and vertical gender segregation; the social construction of women's bodies; and the intrinsic and extrinsic gender segregations, and the questions about the challenges as a society we have to do to advance gender equality and social justice

\section{Presentación}

El siguiente artículo plantea los resultados de una investigación realizada el año 2013, en una empresa minera del norte de Chile, cuyo uno de sus propósitos fue caracterizar, desde una perspectiva de género, a la empresa masculinizada representada en una Minera, ${ }^{1}$ así como la producción y reproducción de estereotipos, roles e identidades hegemónicas de género, que generan brechas, barreras e inequidades para el desempeño laboral de las mujeres principalmente.

La investigación se realizó adoptando una perspectiva cualitativa, llevando a cabo trece entrevistas en profundidad, semiestructuradas, que consideraron ser trabajador/a en la minera como criterio de exclusión, para producir un nivel de homogeneidad entre los/as sujetos/as. Respecto a los criterios de heterogeneidad para la representación de las posiciones de los/as entrevistados/as se consideraron: sexo (hombres, mujeres); áreas o unidades laborales (administración, profesional, operaciones) y tener o no personas a cargo (asociado a posiciones de mayor o menor poder en la organización).

\section{Antecedentes}

\section{a) Participación laboral de las mujeres en Chile}

La participación de las mujeres en la fuerza de trabajo chilena se ha incrementado paulatinamente, en particular desde el año de 1990. A principios de la década de los noventa, la participación laboral de las mujeres era $32,5 \%$, esto quiere decir que de cada diez mujeres en edad de trabajar, poco más de tres, formaban parte de la fuerza de trabajo del país. En el año 2013, el porcentaje asciende a un 45,6\% lo que significa una variación de 13,1 puntos porcentuales de incremento (CASEN, años respectivos).

Si bien ha existido un movimiento de ingreso de las mujeres a los mercados de trabajo, continúan existiendo diferencias significativas en relación con los hombres. De esta manera, para el año 2013, la brecha entre hombres y mujeres respecto de la participación laboral constituye 25,1 puntos porcentuales. Sin embargo, es importante considerar que desde 1990 hasta 2013 la brecha ha disminuido, dado que a comienzos de la década de los 90 esta constituía 41,1 puntos porcentuales, es decir dieciséis puntos porcentuales más que el año 2013.

Entre las mujeres ocupadas predomina el empleo asalariado, pues el 79,1\% de ellas trabaja bajo relación de dependencia, con un empleador y en un régimen salarial, en el año 2013.

El trabajo asalariado se ha mantenido relativamente constante en el tiempo, y tuvo una variación, entre 1990 y 2013, de dos puntos porcentuales. 
Cabe señalar que la brecha salarial bruta ${ }^{2}$ por sexo para el año 2013 , fue de un $33,2 \%$, 0,9 puntos más que la brecha salarial del año 2012 que constituyó un 32,3\% en desmedro de las mujeres (NESI, INE, 2012 y 2013).

También existe una desigual distribución de género en las distintas ramas laborales. Los hombres presentan una mayor concentración, en relación con las mujeres, en las ramas de "explotación de minas y canteras" (92,5\%); "construcción" (93,9\%); "transportes y almacenamiento y comunicaciones" (80,8\%) y "pesca" (90,8\%), mientras que las mujeres presentan mayor concentración en las ramas de "hogares privados con servicio doméstico" (83,1\%), "enseñanza" (72,7\%), "servicios sociales y de salud" (69\%) y en "organizaciones y órganos extraterritoriales" (79\%), en relación con los hombres (NENE, INE, Trimestre móvil marzo- mayo).

La concentración de hombres y mujeres en distintas ramas productivas es generada por la división sexual del trabajo que, según Hartmann, constituye una lógica universal e histórica que ha atribuido roles a hombres -trabajo remunerado- y a mujeres -trabajo de cuidado y doméstico-, de manera jerárquica, lo que provoca que estas últimas queden en la posición de subordinación (Hartmann, 1994). En este contexto, las mujeres se concentran en trabajos asociados a labores de cuidado y educación, como extensión de las labores domésticas tradicionales que, además, son peor remuneradas en los mercados laborales, lo que genera que exista una desigual valoración social frente a estos ámbitos de desarrollo profesional.

\section{b) Participación de las mujeres en el rubro minero}

Chile tiene una de las mayores reservas de cobre en el mundo debido a las condiciones geológicas y a las políticas que incentivan la inversión extranjera en explotación. La reserva mundial de cobre chileno (27,5\%) supera a la reserva de Estados Unidos, México, Perú, entre otros (USGS, 2014).

De acuerdo con el estudio de Díaz , "Mujeres en trabajos de hombres: segregación ocupacional y condiciones laborales en los sectores de la minería y la construcción", la presencia de mujeres en el sector minero ha experimentado un aumento cuantitativamente importante: mientras que en el 2008 eran 9.409 las trabajadoras ocupadas (5,7\%), en el 2012 dicha cifra prácticamente se duplicó, y ascendió a 17.205 trabajadoras, entre empresas mandantes y contratistas de distinto tamaño (Consejo Minero, 2012). Esta cifra corresponde a un 7,2\% del total de trabajadores del sector, y superó en casi un punto porcentual lo observado en 2011 (6,3\%).

Sin embargo, en relación con otros países, Chile se encuentra por debajo de los niveles de incorporación de las mujeres en minería. Por ejemplo, Canadá posee un $16 \%$ de mujeres en el rubro, mientras que en Australia la cifra asciende a un 15,4\% (Díaz, 2014).

Asimismo, es posible establecer que las mujeres se ubican principalmente en labores administrativas, y alcanzan en esta categoría un 88\% de presencia (SERNAM, 2010).

\section{Configuraciones de género en el discurso de hombres y mujeres trabajadores/as en la minera}

\section{a) El ingreso de las mujeres a la Compañía}

En la actualidad la minera en la que se realizó el estudio cuenta con aproximadamente un $8 \%$ de dotación femenina, lo que, si bien la sitúa por sobre el promedio nacional, es bastante bajo en relación con otras ramas productivas del país.
2 Sin considerar horas de trabajo en su cálculo. 
Los motivos que se plantean frente a este bajo porcentaje se relacionan con la formación superior, dado que existe un bajo número de mujeres que estudia carreras relacionadas a la minería, y hacen entrever la existencia de sectores educativos más masculinizados que otros. Este discurso se vincula con lo establecido por la teoría del capital humano de Brown y Corcoran, quienes señalan que el problema está centrado en los incentivos, ya que las mujeres no poseen tantos estímulos como los hombres para invertir en educación y formación para el mercado de trabajo (Brown y Corcoran, 1997).

Se destaca que se ha hecho una búsqueda de trabajadoras para la empresa, lo que ha sido difícil ya que no existen perfiles de mantención u operación. Los requieren entre tres y cuatro años de estudios técnicos.

También se señaló que existían algunas áreas de preferencia para las trabajadoras, ya que les permitían compatibilizar labores de cuidado, trabajo doméstico y responsabilidades familiares, es decir, atender a una estructura de roles en función de la división sexual del trabajo (Hartmann, 1994) que, históricamente, las ha situado en este tipo de labores. Estas áreas son principalmente de carácter administrativo, en las que desempeñan jornadas laborales en horario "de oficina". Sin embargo, existen algunas áreas que rechazan la presencia de mujeres en la selección, lo que se vincula con las restricciones extrínsecas señaladas por Kabeer (2012) donde, entre otros elementos, se reflejan en las preferencias de los/as empleadores/as; normas culturales y regulaciones legales que establecen el acceso y control de recursos (tierras, capital, crédito, tecnología, etc.) que constituyen desventajas para las mujeres. Lo anterior se ve reflejado en el discurso de la encargada de selección de personal de la minera, quien visualiza las dificultades para insertar a mujeres en determinadas áreas donde, de antemano, existe un rechazo:

"Hay áreas a las que no les gustan las mujeres, tal área nunca recibe mujeres, o no les gusta las mujeres, siempre que les llevo mujeres las rechazan (...)". (Mujer, administración, sin personas a cargo).

\section{Las primeras mujeres en Operaciones Mina}

El área de Operaciones Mina había estado conformada preferentemente por hombres, responsables de manejar maquinaria pesada para transportar material minero, además de trasladar cables eléctricos de gran peso. Paulatinamente se han integrado mujeres, quienes, según los/as entrevistados/as, se han desenvuelto a la par con sus compañeros de trabajo.

En el relato de las mujeres de esta unidad se plantea que inicialmente el lugar y el trabajo les habían sido diferentes en relación con lo que habían conocido anteriormente. En este sentido, para ellas ha sido un gran desafío.

Varias de las trabajadoras que ingresaron con el incentivo de reclutamiento de la minera, continúan trabajando, y, pese a la cultura machista existente, han logrado validarse:

"Y entré a trabajar acá, también fui de las primeras mujeres que entraron a Operaciones Minas (...) no había mujeres, y fuimos ocho las que llegamos a trabajar acá. No tenía idea lo que era operar un camión, no conocía los camiones grandes, con suerte lo más grande que había manejado era una camioneta o algo así y llegué acá, bueno acá nos enseñaron de todo, la capacitación fue muy completa y muy buena (...) Bueno, y trabajar en sí, como te digo, igual fue un poquito difícil al principio. Generalmente la mayoría de los compañeros nos recibieron bien. Sí hay siempre, hay un poquito, que eran más machistas: que la mujer en la mina, que 
no que... pero yo creo que al final igual les doblegamos la mano, y nos aceptaron igual”. (Mujer, Operaciones, sin personas a cargo).

También, a través del relato de las mujeres de Operaciones Mina, emerge la disposición de realizar cualquier tipo de trabajo, de estar dispuestas a enfrentar los nuevos desafíos:

"Voy a donde me manden, me gusta, o sea las mujeres no estamos muy hechas para esos equipos pesados porque hay que tener punchi... para algunos porque son medio complicados, tienen frenos... nos han dicho que son pesados. No hay nada que no podamos hacer... es una carrera... “. (Mujer, Operaciones, sin personas a cargo).

En este contexto, la incorporación de mujeres en Operaciones Minas constituye un avance en términos de romper con barreras de ingreso de las mujeres en espacios masculinizados donde existen labores que tradicionalmente que habían sido consideradas para hombres, rompiendo así inicialmente con las segregaciones horizontales y con los estereotipos negativos que establecen que las mujeres "no están hechas para el trabajo duro".

Sin embargo, es necesario poner en tensión estas aseveraciones ya que el ingreso de mujeres no pareciera ser un mérito individual o colectivo, en la medida que existen estrategias en el campo de la minería, asociadas a equipamiento de fácil utilización dirigido justamente a trabajadoras de este rubro. Asimismo, la pregunta que emerge es si este ingreso es en pos de una autonomía (Lagarde, 1992) -más allá de una autonomía económica-, que permite a las mujeres auto determinar sus decisiones de manera libre, o bien, es un discurso establecido que encierra una forma de dominación por parte de la empresa para generar un plusvalor productivo.

También es importante señalar que esta incorporación no ha significado el ascenso de las trabajadoras a puestos de mayor jerarquía, ya que son muy pocas las que se desempeñan como supervisoras.

Así, se devela la existencia de una segregación vertical (Díaz, 2014) sustentada por estereotipos negativos (Anker y Hein, 1986), vinculada a que las mujeres acatan mejor las órdenes y siguen más fielmente los protocolos, juicio que podría ser contraproducente al momento de ubicar a mujeres en tipos de trabajo más autónomos, como es el de la supervisión.

\section{Posibilidad de ascenso social}

Se percibe el trabajo en la minera como una oportunidad de ascenso social por medio de mejores remuneraciones, y supera la existencia, para las trabajadoras, de un horizonte de desarrollo profesional y económico acotado:

"Chicas que estaban en sus casas, egresadas de cuarto medio... yo les decía que tenían el norte puesto en Chañaral... de repente tuvieron la oportunidad de trabajar en una empresa minera más o menos grande, tener buen ingreso, poder adquirir bienes materiales, por ejemplo, su casa, su auto". (Mujer, administración, sin personas a cargo).

Desde las jefaturas también existe un reconocimiento sobre lo que ha significado para las mujeres desempeñarse en el ámbito minero y se destaca el rol que cumplen muchas de ellas como principales proveedoras de sus familias. 
3 Utilizaremos el concepto de clase social sin profundizar en el debate de este concepto en la actualidad. Sin embargo destacamos la noción de habitus de clase de Bourdieu que remite a "el conjunto de agentes que ocupan posiciones semejantes y que, situados en condicionamientos semejantes y sometidos a condicionamientos semejantes, tienen todas las probabilidades de tener disposiciones e intereses semejantes y de producir por anto, prácticas y tomas de posiciones semejantes" (Bourdieu, 1990: 284).
Los discursos de trabajadoras con personas a cargo, que han accedido a estudios superiores, dejan entrever una distancia frente a las experiencias de vida y en los modos de desenvolverse en el trabajo respecto de las trabajadoras operarias. Inicialmente, no se especifica ni se detallan estas diferencias, pero sí se establece el concepto de "otros mundos", lo que es de interés, dado que en la minería, por el número y la diversidad de trabajadores/as, se puede apreciar que es un espacio micro social que establece distinciones entre experiencias de vida. Esto se ve reforzado por la siguiente cita:

Esta distinción de clase social ${ }^{3}$ se observa como una discriminación desde el relato. Se plantea que las mujeres de Operaciones Mina, por tener una menor formación y por provenir de una clase social más baja, ingresarían al trabajo con intenciones de conseguir "otras cosas" que van más allá de la propia labor cotidiana. Así, entre líneas se lee que el ingreso ha incidido en el "desarme de familias" a través del desarrollo de relaciones amorosas con sus compañeros de trabajo, e implica la generación de conflicto entre parejas preestablecidas. En el relato, al señalar "vienen a buscar algo más", pareciera hacer referencia a un mecanismo de escalada social vinculado una mayor posición de prestigio por medio de relaciones amorosas/sexuales:

“Sí, bien, pero acá han estado contratando mujeres como operadores, que de repente pueden pensar como distinto... a ver cómo te explico, como que chacrean la cuestión... Porque creen que no sé oh, que por lo que he visto, vienen así como a buscar algo. No es que esté hablando mal de ellas, ni nada, pero es que como son personas menos preparadas, a lo mejor su educación todo, ya acá, yo entro, y busco algo más... no sé, no sé, pero, pero porque se han desarmado familias, poh, por la llegada de las niñas". (Mujer, control mineral, con personas a cargo).

La discriminación visualizada anteriormente da cuenta de un sistema de restricciones proveniente de las mujeres profesionales hacia mujeres operarias, y tiene como origen la clase social y el género dado que pre instala en las mujeres operarias ciertas conductas que son menos valoradas socialmente y que no responden a la feminidad hegemónica de una estructura patriarcal.

\section{Cambios y resistencias masculinas}

Al momento de ingreso de las mujeres en Operaciones Mina, existía una cultura machista plasmada en los modos de expresión de los hombres a través de bromas y tallas, que dejaban a las mujeres al margen, lo que se conecta con la construcción de la masculinidad hegemónica (Olavarría, 2001a y b; Salguero, 2007), en la necesidad de constituirse como hombre a través del reconocimiento de otros/as y a través de la generación de cierres y aperturas de grupos sociales, en el sentido de pertenecer o no pertenecer al grupo de los hombres.

Las actitudes que se identifican como propias de un ambiente masculinizado tienen que ver con relaciones de menor respeto/reconocimiento de un/a otro/a legítimo/a (Maturana y Zöller, 1993) y de poco autocuidado, representado a través de la imagen en el poco cuidado del cuerpo (desordenados, cochinos y hediondos), que comienza a transformarse en la medida que las mujeres ingresan a esta unidad. Así, el cuerpo y su expresión se modifican por medio de la autorregulación a través de la intervención femenina, lo que queda reflejado en lo siguiente: 


\begin{abstract}
“Hubo que hacer reuniones con los trabajadores para promover el respeto, aunque sabes tú que el hecho de que trabajen mujeres en el área se suavizan ellos, se modera el vocabulario, el trato, se portan mejor, son más ordenaditos, son más limpiecitos. Cuando no hay mujeres son así no más, desordenados cochinos y hediondos. Entonces la presencia femenina hace que ellos se regulen, cambia, por ejemplo ellos, el equipo es enorme, el camión por ejemplo es como subirte a una casa de tres pisos, entonces ellos se preocupan de dejar limpiecito si saben que viene detrás de ellos una mujer que opera su mismo equipo (...) entonces si saben que viene una mujer, se preocupan de poner silicona, desodorante ambiental". (Mujer, administración, sin personas a cargo).
\end{abstract}

Es interesante destacar que en todos los discursos se valora la presencia de las mujeres argumentando que, a través de estas, se logran moderar actitudes y discursos, lo que también se vincula a que dichos espacios se permeen con una mayor profesionalización que, a través de un clima laboral adecuado, permite una mayor productividad. Lo anterior se ve reflejado en la siguiente cita:

“Por ejemplo, en las reuniones también la actitud cambia. En el tiempo que yo llegué eran bien agresivos. Mirá, conmigo nunca fueron mal educados, me trataban con respeto, pero su actitud era como de combate, estaban muy presentes los dirigentes sindicales”. (Mujer, administración, sin personas a cargo).

En un inicio, desde la percepción de los hombres que trabajaban en Operaciones Minas, también existió un rechazo ante la presencia de mujeres, y ellos lo interpretaban como la pérdida de libertad para expresarse. Así, la presencia/ausencia de actores en una determinada área, atravesado por identidades hegemónicas de género (Lagarde, 1992; Olavarría, 2001a y b; Salguero, 2007) implica modificaciones en conductas, discursos y formas de presentación del cuerpo. En este sentido, los hombres realizan una performance al momento de enfrentarse a sus compañeras mujeres, que tiene relación con modificar conductas provenientes de una cultura que responde a una masculinidad hegemónica, en la que existen prácticas y discursos específicos de validación en el entorno y frente a sus pares (Butler, 1990). Esta performance es vivida por los hombres como una "pérdida de libertad", dado que produce un cambio en sus conductas, modos de relación y razonamiento, que los aleja de las estructuras conocidas de una masculinidad hegemónica. Lo anterior se justifica a través de la siguiente cita:

"Me enteré de las mujeres cuando iban a entrar porque los varones comentaban afuera que las mujeres, no era lo mismo, como que también ellos pierden libertad de poder expresarse libremente, ya tienen que cuidar su vocabulario, ese tipo de cosas". (Mujer, administración, sin personas a cargo).

\title{
b) Estereotipos femeninos y masculinos en el ejercicio del cargo
}

En el discurso de las mujeres se señalan diferentes posibilidades para ejercer el cargo debido a los procesos de socialización primarios y secundarios diferenciados por roles de género (De Barbieri, 1993; Kabeer, 2012). De este modo se plantea que para los hombres sería más cercano el trabajo con maquinaria pesada dado que en su socialización primaria atendería a generar una familiaridad con este tipo de elementos, lo que se ve reflejado en el siguiente juicio de una trabajadora:

“Es que el hombre tiene desarrollado de chico el gusto con los camiones, autos y como que tiene esa habilidad innata, nosotras la tenemos que desarrollar. Ellos aprenden de antes, nos ganan. Nosotras nacemos con eso... con la pinturita... entonces ahí nos llevan ventaja”. (Mujer, Operaciones, sin personas a cargo). 
Existe una percepción positiva sobre el trabajo de las mujeres en la minería, sustentado en un tipo específico de labores protocolizadas, en las que es necesario seguir órdenes y etapas específicas para el trabajo, y se deja entrever que los hombres, respondiendo a la construcción de una masculinidad hegemónica, improvisarían en mayor medida en sus labores, dado que manejan el trabajo cotidiano. En este sentido, serían más "innovadores" u "osados", características que no son valoradas positivamente atendiendo a la necesidad de continuar protocolos de seguridad establecidos para el manejo de maquinaria pesada.

"Nosotros, los hombres, somos, a ver, cómo explicarte, somos un poco desordenados, en el sentido de que partimos muy bien, pero al poco andar, de repente nos empezamos a creer el cuento, entonces cuando tu partes en un trabajo de tanta responsabilidad como conducir un camión que transporta 8500 toneladas, es súper delicado tu entorno y los procedimientos (...) en cambio la mujer no, hemos descubierto que, de alguna forma, nosotros les hemos entregado las herramientas y procedimiento y lo siguen al pie de la letra, es muy responsable y maneja según la letra eso genera una muy buena operadora (...) son bien consistentes en sus trabajos, son ordenadas, cumplen". (Hombre, operaciones, con personas a cargo).

Al remitirnos a las características valoradas del trabajo de las mujeres, en el discurso se plantea una naturalización de estas habilidades, sustentada por ser más cuidadosas, promover más la protección, lo que tendría su raigambre en la tarea histórica de cuidar a los/as hijos/as. Asimismo se devela la performatividad de roles, que bajo una estructura patriarcal y capitalista instalan la figura e impronta de la mujer modelo, y conjugan el recato, el cuidado y la productividad. Así, se visualiza la existencia de "cuerpos dóciles" que, según lo planteado por Foucault , gestados por medio de una dominación implícita en el ámbito laboral, desde jefaturas a subordinados/as, que recae sobre los cuerpos (Focault, 2002).

\section{c) Compatibilidad/incompatibilidad del trabajo remunerado con labores de cuidado}

Las labores del cuidado fueron señaladas como una barrera de permanencia en la minería y constituyen, según Kabeer (2012), restricciones intrínsecas a la participación laboral de las mujeres. Cabe destacar que esta percepción se instala bajo un modelo capitalista que otorga mayor valor social al trabajo remunerado que al trabajo no remunerado, de cuidado y doméstico.

A través del discurso se señala cómo, para continuar con el ritmo de la rutina diaria, se vuelve necesario descansar menos, es decir, generar una soportabilidad social que remite a un "conjunto de prácticas hechas cuerpo que se orientan a la evitación sistemática del conflicto social (...) permiten la aceptación, por parte del sujeto y la sociedad toda, de que la vida social "se-hace"como un-siempre-así"(Scribano, 2009: 145), para la producción en el trabajo con un mayor gasto energético y sin generación de cuestionamientos incidiendo directamente en el trabajo reproductivo, lo que queda descrito en la siguiente cita:

"Porque cuando tenemos hijos parece que nos acostumbramos a dormir menos, yo te digo duermo y estoy pendiente... tiene activado ese sexto sentido de "qué pasa" y en el fin de semana estoy más relajada”. (Mujer, Operaciones, sin personas a cargo).

De este modo, emerge en el discurso un "cuerpo resistente" vinculado al soporte de tareas atribuidas socialmente a los sujetos, en el marco de los roles de género, junto a nuevas tareas del trabajo remunerado, produciendo así la configuración de elementos 
relacionados a mecanismos de soportabilidad social, de soportabilidad de los cuerpos y mecanismos de regulación de emociones (Scribano, 2009).

En el discurso de las mujeres se demuestra una incompatibilidad con ser madre, cumplir con labores de cuidado y trabajar en la minera, lo que se refleja a través de las dificultades para pedir permisos. El "no se ve bien" al momento de pedir permiso a las jefaturas para cumplir actividades familiares en horario de trabajo, demuestra cómo, de manera implícita, se permiten o se inhiben ciertas prácticas.

\section{Cercanía/lejanía espacial}

También, se destacan como restricciones los traslados y las distancias que separan la minera del hogar. En este caso existen posiciones encontradas dado que, por una parte, se valora que la empresa se encuentre cerca de la ciudad y que no existan sistemas de turnos prolongados, pero también se destaca que la distancia de la minera con la ciudad genera dificultades para desplazarse en caso de alguna emergencia en el ámbito doméstico/familiar.

\section{Apoyo en el cuidado}

Para compatibilizar el trabajo remunerado con el no remunerado, es frecuente que las mujeres recurran a otras mujeres: amigas, familiares, asesoras del hogar, etcétera, quienes apoyan en el trabajo doméstico y de cuidado y permiten a las primeras tener horas de descanso y/o de trabajo.

También se planteó la necesidad de cubrir las labores de cuidado y de trabajo doméstico por medio de una asesora del hogar puertas adentro, es decir, una mujer (sobre el $95 \%$ de los casos según NENE, INE, 2015) que cumple el rol de cuidadora principal. Así, se destaca cómo los hijos/as adoptan comportamientos similares a los de la persona que trabaja cuidándolos, relacionado a lo mencionado por Hoschild (2008) sobre cómo en las sociedades capitalistas, que están a disposición de la producción económica, generan una suerte de traspaso de afectos, los que también son mercantilizados, en la medida que madres y padres tienen que pasar largas jornadas de trabajo, mientras que otras personas, cuidan a sus hijos/as. Dichas personas son en su mayoría migrantes de los países colindantes con la zona norte de Chile, principalmente de procedencia boliviana, peruana, e indígena de la zona altiplánica (Menara y Garcés, 2012: 5-34), quienes, al mismo tiempo, tienen a sus hijos/as con otras personas a su cargo, en su país de origen.

Lo anterior da cuenta del cruce existente entre clase social, género y migración, donde finalmente estas mujeres se encuentran en posiciones socialmente desventajosas.

\section{d) Segregación vertical, las barreras para ascender}

La segregación vertical es uno de los elementos que imposibilitan la llegada de las mujeres a cargos de mayor jerarquía, y constituye barreras que permean relaciones, prácticas, actitudes y motivaciones, además de fomentar prejuicios que producen que un menor número de mujeres alcancen puestos elevados en la estructura organizacional. Al respecto, Abramo señala que uno de los supuestos que fundamenta las decisiones de no ascenso tiene que ver con el prejuicio de que las mujeres son segundas proveedoras de la economía de sus hogares (Abramo, 2004).

En el discurso de las trabajadoras se reconoce la inexistencia de mujeres en cargos de jefatura, avalado por uno de los gerentes del ámbito de la minera, quien señala que desconoce los motivos de esta situación. 
Considerando la historia de la minera, se plantea que existió una mujer gerenta, quien se sometió a un proceso de validación frente a los/as trabajadores/as, quienes de antemano ponían en tela de juicio sus capacidades. Se destaca la actitud directa para plantear las dificultades que emergían en el trabajo cotidiano como una virtud valorada en el trabajo y para la dirección de equipos. En este caso, surge el cuestionamiento sobre si los hombres tienen que validarse al igual que esta mujer para ocupar el cargo de gerenta. Pareciera ser que, cuando un hombre es embestido en un cargo, se da por hecho de que es competente para tales funciones cuestión que no resulta habitual para el caso de las mujeres.

También, es importante señalar que la mujer gerenta a la que se hace referencia, era extranjera, estadounidense, cuestión planteada como facilitador para validarse en la empresa, atravesado por una estructura de clases y en la que, a determinados/as extranjeros/as -excluyendo a los bolivianos/as, peruanos/as, colombianos/as, haitianos/as, etcétera- se los valora mayormente por el aspecto físico. De este modo, la procedencia étnica permite posicionarse, en este caso, aventajadamente frente a las demás mujeres, generando así una incidencia entre la figura con poder y el colectivo de trabajadoras.

Se planteó que la figura de secretaria es una jefatura implícita, en la medida en que ocupa un rol que permite estructurar el trabajo de los/as demás. Así, se destaca que la persona que ocupa este cargo en una gerencia de la minera, está a cargo de distintos proyectos y organiza los grupos de trabajo para mantener el avance y el funcionamiento del área. Asimismo, las labores tradicionales de la ocupación de secretaria, tales como servir el café, no son realizadas por esta mujer, dado que se ha ganado un espacio de confianza y responsabilidad lo que ha llevado también a los hombres de dicha gerencia tener autonomía sobre estas labores.

Esto resulta interesante dado que, además de reconocer las funciones que desarrolla esta persona, se asemeja con lo que sucede en el espacio doméstico, en el que las mujeres son quienes dictan las normas y establecen los procedimientos dentro del hogar, y son valoradas en la medida en que se circunscriben en este espacio privado, mientras que los hombres cumplen con roles de proveedores económicos, además de ejercer un poder público. Asimismo, la secretaria tiene un cierto poder en la medida en que su jefatura se le otorga y le permite el funcionamiento, lo que puede constituir el símil con lo que ocurre en los hogares tradicionales, en los que el poder es otorgado por el "jefe de hogar", que es la figura del proveedor principal. En este sentido, si bien la secretaria cumple un rol fundamental en el área, su poder no es reconocido materialmente en la medida en que no ocupa realmente un cargo de jefatura, sino que solo ejerce un reconocimiento simbólico que da cuenta del no reconocimiento real de las labores que lleva a cabo.

En el discurso de una trabajadora que tiene personas a su cargo en el área de Operaciones Mina, se destaca un liderazgo relacionado con establecer límites claros frente a las posibilidades de acción de los/as trabajadores/as, que son actualizados permanentemente para evitar el exceso de permisos.

En este caso existe una performance referida al ejercicio de poder vertical, desde la mujer hacia los/as demás trabajadores/as, estrategia considerada como necesaria para validarse y "hacerse respetar" en el trabajo, lo que podemos conectar con el concepto de 'interaccionismo estratégico', de Goffman, debido a que se desarrolla un modo de presentarse para lograr los objetivos (Goffman, 2006). Dicha estrategia se asocia a un ejercicio de un poder masculinizado en términos hegemónicos (Olavarría, 2001a y b; Salguero, 2007), que da cuenta permanentemente de la asimetría de posiciones que "pone en su lugar" a los/as trabajadores/as subalternos/as. Este "poner en el 
lugar" es necesario para mantener el orden de la estructura de laboral, pero también deja entrever, el temor de perder el poder que se ha ganado, tratando de atesorarlo de manera individual. Lo anterior se puede expresar por medio de la siguiente cita:

"Hay que ahí rayarles la cancha, no, o sea no porque sea mujer, sea joven, te respetan, todo, pero hay viejitos más tozudos que tú teníh que saber manejarlos, porque ellos se aprovechan de la forma de pedir los permisos, pedir vacaciones, y tú teníh que pararles la... rayarles la cancha, porque si tú les das mucha ya... todos permisos, ya, que llegue un poquito más tarde, se suben todos por el chorro. Entonces hay que rayarles la cancha del principio... y recordarles cada cierto tiempo de nuevo". (Mujer, control mineral, con personas a cargo).

\section{Conclusiones}

Las conclusiones y desafíos de la investigación remiten a que el ingreso y permanencia de las mujeres a la minera no ha estado exento de tensiones y dificultades. Por una parte, dicho ingreso ha producido modificación de prácticas y significaciones, y ha implicado una valoración de dichas transformaciones en términos productivos. Asimismo, las estrategias de las grandes empresas, además de las herramientas utilizadas -por ejemplo empresas que diseñan camiones de fácil uso-, han permitido el ingreso de las mujeres a la minería, lo que se traduce en la necesidad de mayor fuerza productiva en el marco de un sistema capitalista.

Un trabajo es masculinizado, no solo por la alta presencia de hombres sino por sus prácticas. En esta línea se planteó que en la empresa, en particular la unidad de Operaciones Mina, los hombres que trabajaban solían adoptar prácticas discriminatorias frente a las demás personas, en el sentido de "poner al otro/a en su lugar" estableciendo posibilidades de apertura y cierre en los grupos de varones, conjugado con estéticas corporales de poco cuidado personal y una disposición de "combate" -en palabra de los/as entrevistados/as- frente a los requerimientos de las jefaturas.

Se destacó que este escenario se ha modificado con el ingreso de trabajadoras a la organización, quienes han gestado nuevas interacciones, y más dinámicas. De este modo, se valoraron elementos atribuidos a las mujeres como la responsabilidad y el acatar órdenes, mediante la implicación de la visibilización de "cuerpos dóciles". También emergen "cuerpos resistentes" de mujeres, que deben soportar diversas tareas atribuidas socialmente en el marco de los roles de género, junto a nuevas tareas del trabajo remunerado, produciendo así la configuración de elementos relacionados a mecanismos de soportabilidad de los cuerpos y mecanismos de regulación de emociones (Scribano, 2009).

También, se visualizaron segregaciones horizontales y verticales, que dan cuenta de una organización jerárquica en la que se producen barreras que impiden a las mujeres desempeñarse en cargos de mayor jerarquía. Frente a lo anterior, las mujeres adoptan determinadas performances y estrategias para ser reconocidas y validadas por sus pares y superiores (Butler, 1990; Goffman, 2006).

Finalmente, existen elementos conectados a la división sexual del trabajo y a las labores de trabajo no remunerado (doméstico y de cuidado), como elementos que también se estructuran como barreras para el desempeño laboral.

Como ideas fuerzas de las conclusiones se puede establecer lo siguiente: 
a) Los lugares de trabajo se encuentran permeados por lógicas capitalistas y de estructuras patriarcales que los configuran, e inciden en prácticas, identidades y roles de los/as sujetos. En este marco, las mujeres resultan en mayor medida oprimidas porque se enfrentan a brechas, barreras e inequidades de género configuradas en torno a un mandato de maximización de la producción.

b) En el contexto actual -bajo la estructura patriarcal y capitalista- los cuerpos de las mujeres se encuentran oprimidos, y son producidos y reproducidos de manera sumisa y dócil en los lugares de trabajo. Asimismo existen cuerpos resistentes de las trabajadoras, quienes soportan las condiciones y se sobre esfuerzan produciendo un mayor gasto energético. Ambos cuerpos se producen, reproducen y al mismo tiempo estructuran mecanismos de soportabilidad corporal y mecanismos de regulación de las emociones que permiten la oclusión de los conflictos y las contradicciones sociales. La pregunta que nos planteamos es cómo en este marco podemos propender a la existencia de cuerpos libres, que se despojen de ataduras de dominación de las estructuras mencionadas.

c) Las restricciones intrínsecas a la participación laboral de las mujeres se reproducen por los estereotipos de género, tanto de hombres como de mujeres, transversales a las posiciones que ocupan los/as sujetos/as en la estructura laboral (personas que tienen trabajadores/as o no a su cargo), e imposibilitan su validación laboral, tanto como jefaturas como para aquellas mujeres que ocupan otras posiciones. En este sentido, una pregunta que queda pendiente para futuros estudios es conocer cómo se pueden eliminar estos estereotipos para comprender al/a la otro/a de una manera legítima.

d) Como restricción extrínseca, y asociado a barreras a la participación de las mujeres, se encuentra el congeniar labores de cuidado y de trabajo remunerado, lo que tiene fuerte vinculación a los roles de género en función de una división sexual del trabajo que tiene larga data en nuestras sociedades. De este modo, las mujeres deben adoptar estrategias para el cuidado de sus/ as hijos/as a través de redes familiares o accediendo al pago monetario de personas que hacen esta labor.

La última pregunta que nos cabe en estas conclusiones tienen que ver con cómo es posible modificar estos roles para que, tanto mujeres como hombres, puedan acceder al cuidado (que es necesario para el desarrollo subjetivo de cada persona) y al ingreso monetario (que permite la autonomía económica). De esta manera, nos cuestionamos a qué sociedad queremos aspirar, ¿una sociedad en la que el trabajo remunerado en el espacio público se encuentra sobrevalorado en relación con el trabajo no remunerado de cuidado?, o ¿una sociedad más justa, en la quelos sujetos puedan ser actores que modelen sus roles en función del bienestar de ellos/as y de los/as demás? 


\section{Bibliografía}

》 Abramo, L. (2004). “¿Inserción de las mujeres en América Latina: Una fuerza de trabajo secundaria?”. En Estudios Feministas, No. 12. Florianópolis, Brasil.

» Anker, R. y Hein, C. (1986). Sex Inequalities in Urban Employment in the Third World. Sto Martin's Press. Nueva York, Estados Unidos.

"Bourdieu, P. (1990). Espacio social y génesis de las clases. México, Sociología y cultura.

» Brown, C. y Corcoran. M. (1997). Sex- based differences in school content and the male-female wage gap. Journal of labor Economics 15.

» Butler, J. (1990). El género en disputa. El feminismo y la subversión de la identidad. Barcelona, Paidós.

»Consejo Minero (2012-2013). Reporte anual. Disponible en <http://www.consejominero.cl/wp-content/uploads/2013/o9/Reporte-Anual-CM-2012-2013.pdf>.

"Díaz, E. (2014). Mujeres en trabajos de hombres: segregación ocupacional y condiciones laborales en los sectores de la minería y la construcción. Chile, Dirección del Trabajo.

» Barbieri T., De. (1993). “Sobre la categoría de género. Una introducción teórico- metodológica". En Debates en Sociología, No. 18. Pontificia Universidad Católica del Perú. Perú, Departamento de Ciencias Sociales.

»Foucault, M. (2002). Vigilar y castigar: nacimiento de la prisión. Buenos Aires, Siglo XXI.

» Goffman, E. (2006). La presentación de la persona en la vida cotidiana. Buenos Aires, Amorrortu..

» Hartmann, H. (1994). “Capitalismo, patriarcado y segregación de los empleos por sexos”. En Borderías, C., Carrasco, C., Alemany, C. (Compiladores), Las mujeres y el trabajo. Rupturas conceptuales, , Barcelona, Fuhem/Icaria.

» Hochschild, A. (2008). La mercantilización de la vida íntima. Apuntes de la casa y el trabajo. Madrid, Katz.

"Instituto Nacional de Estadísticas INE. (2015). Nueva Encuesta Nacional de Empleo NENE. Trimestre móvil Marzo- Mayo, 2015. Chile.

» Instituto Nacional de Estadísticas INE (2013). Nueva Encuesta Suplementaria de Ingreso NESI. Chile.

»Lagarde, M. (1992). Antropología de los cautiverios: Madresposa, monjas, putas, presas y locas.México, Universidad Autónoma de México.

"Lerner, G. (1990). La creación del patriarcado. Nueva York,Crítica.

" Maturana, H. y Verden Zoller, G. (1993). Amor y Juego. Fundamentos olvidados de lo humano. Desde el patriarcado a la democracia. Santiago, Editorial Instituto de Terapia Cognitiva.

» Menara, H y Garcés, A. (2012). Mujeres peruanas en las regiones del norte de Chile: Apuntes preliminares para la investigación. Estudios Atacameños № 44 / 2012, pp. 5-34. Arqueología y Antropología Surandinas. 
» Ministerio de Desarrollo Social (Versiones: 1990- 2013) Encuesta de Caracterización Socioeconómica Nacional, Casen, Chile.

»Olavarría J. (2001a). ¿Hombres a la deriva? Poder, trabajo y sexo. Chile, FLACSO.

- (2001b). Hombres: Identidad/es y violencia. Segundo encuentro de estudios de masculinidades: Identidades, cuerpos, violencia y políticas públicas. Chile, FLACSO.

» Oyarzún, K. (2014). “Enfoque de género en la aplicación de programas y políticas públicas: gestión y transversalizaciones". Chile, Documento de Trabajo Interno. Servicio Nacional de la Mujer.

"Salguero, A. (2007). "El significado del trabajo en las identidades masculinas". En Jiménez y Tena (coordinadoras), Reflexiones sobre masculinidades y empleo. Universidad Autónoma de México. Centro Regional de Investigaciones Multidisciplinarias, México.

»Scribano, A. (2009). “A modo de epílogo. ¿Por qué una mirada sociológica de los cuerpos y las emociones?”. En Figari C. y Scribano A. (compiladores), Cuerpo(s), subjetividad(es) y conflicto(s). Hacia una sociología de los cuerpos y las emociones desde Latinoamérica Buenos Aires, Fundación Ciccus.

"Servicio Nacional de la Mujer, SERNAM. (2010), “Demanda de mano de obra calificada en proyectos de minería y energía”. Montecinos C, MultiTaskBrokers Chile Ltda. Chile. 